\title{
Barriers to COVID-19 Containment: The Role of Vulnerable Populations
}

\author{
Habib $\mathrm{AK}^{1}$ and Habib $\mathrm{KJ}^{2 *}$ \\ ${ }^{1}$ Parkview Heart Institute, Indiana, USA \\ ${ }^{2}$ Materials Science and Photo-Electronic Lab., RE Program, EBR Center, KISR, Kuwait
}

Editorial

Volume 5 Special Issue 1

Received Date: August 24, 2020

Published Date: September 01, 2020

*Corresponding author: Habib KJ, Materials Science and Photo-Electronic Lab., RE Program, EBR Center, KISR, P.O. Box 24885 SAFAT, 13109, Kuwait, Email: khaledhabib@usa.net

\section{Editorial}

Containment of the coronavirus pandemic of 2019 (COVID-19) has initially surrounded vulnerable population such as the elderly and patients of low socioeconomic status [1]. The infection rate has been unacceptably high throughout the world however more so in developed countries such as the United States of America (USA), Brazil, United Kingdom (UK), Italy, Spain and France [2] despite the presence of advanced medical services in those countries. This trend is especially seen in patients of poor socioeconomic status. Several questions have been raised such as why some countries have been able to control their infection rate and containment [3]. Few nations have resumed normalcy in this global pandemic crisis. Among many answers of those questions can be found in the welfare of vulnerable populations. Health status and comorbid conditions play an important part with several factors leading to poorer outcomes such as obesity [4], respiratory illnesses that disproportionately affect the poor and elderly and the lack of nursing care in the outpatient and inpatient hospital setting [5]. Rather than the sole emphasis on personal protective equipment (PPE) and social isolation, which is necessary for the containment of a pandemic, the care of vulnerable population will ultimately lead into less spread and improved morbidity/mortality of this disease. Addressing the comorbid and socioeconomic conditions of vulnerable populations would lead to more containment and less spread. The fears of providing these population with the required healthcare may inadvertently lead to the increase spread of disease and isolation of these populations. While a vaccine is being developed, we must be cognizant of the barriers that separate vulnerable populations may also increase the spread of COVID-19 to all populations. However at this moment, without a vaccine and unreliable herd immunity, care for the most vulnerable is the only weapon available against COVID-19 that does not involve a physical or social barrier, however in most societies that may be the main impediment in its containment.

\section{References}

1. Rui L (2020) Basic Understanding and Perspectives for Coronavirus. Nanomedicine \& Nanotechnology Open Access 5(1): 1-2.

2. Anusiem CA (2020) Covid-19 Pandemic and Its Novel Virus: Lessons Learnt so Far. Virology \& Immunology Journal 4(1): 1-3.

3. Coronavirus map.

4. Cucin RL (2020) Reducing Covid-19 and Future Pandemic Mortality by Eliminating Obesity Comorbidity. International Journal of Surgery \& Surgical Techniques $4(1): 1-2$.

5. Lin Z, Yan G, Ping Y, Yuanhao W, Jingjing G, et al. (2020) Nursing Care of Critically Ill Patient Infected With COVID-19 Virus in China, Shanghai: A Case Report. Nursing \& Healthcare International Journal 4(1): 1-5.

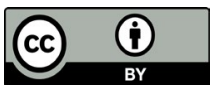

